\title{
A Review and Comprehensive Study of Wireless Channel in Mobile Communication System: Fading Phenomena and Estimation
}

\author{
Awwab Q. Jumaah ${ }^{a *}$ \\ ${ }^{a}$ University of Babylon, Collage of Engineering, Department of Electrical Engineering, Babil, Iraq.
}

\begin{tabular}{l} 
A R T I C L E I N F O \\
\hline Article history: \\
Received 03 February 2019 \\
Received in revised form 25 \\
Accepted 28 March 2019 \\
\hline Keywords: \\
Fading channels \\
Channel state information \\
Channel impulse response \\
Estimation techniques \\
Second order statistics
\end{tabular}

Second order statistics

\begin{abstract}
A B S T R A C T
In wireless communication systems, the channel estimation problem has been played an essential challenge to accurately retrieve the channel state information (CSI) such that reliable communication \& wide coverage can be provided. Due to the improvement and rapid growth of communication systems and in order to maintain a reliable data transmission, estimation of CSI has become necessary. This in turn results, precise receiver demodulation, accurate decoding, and equalization processes. This paper gives a survey on a fading phenomena and a comprehensive review of the recent works that have already been done and studied related to the problem of estimating channel parameters in wireless communication systems. Varieties of best channel estimation techniques that have been recently evolved are explored. Comparison between them in terms of computational cost, simplicity and appropriateness conditions is also discussed. This paper also provides a basic introduction of wireless channel model, SIMO and MIMO channel.
\end{abstract}

(C) 2019 University of Al-Qadisiyah. All rights reserved.

\section{Introduction}

Signals transmitted through a wireless communication channel exposed to a severe physical environment in a complicated manner. Obstructions such as mountains, infrastructures, and trees produce signal diffractions, reflections and scattering. Subsequently at the receiver end, received signals have falsifications, delays, interference and different phase shifts due to multipath signals generated. The term fading comes from devastatingly interfering multipath signals with each other. The fading might affect the quality and reliability of communication systems by causing decay in a power signal to noise ratio (SNR), which may result in a communication failure. The phenomena of Fading can be categorized into two basic kinds: small-scale fading and large-scale fading [1]. Small-scale fading is also known as a Rayleigh fading which comes from variations in phase and amplitude of transmitted signals since there are trivial spatial separation alterations among a transmitter and receiver. A Rayleigh pdf (probability density function) can statistically describe the envelope of received signals when there is fading, no a line of sight, signal component presents whereas a Rician pdf can describe a small fading envelope if there is no dominant fading present [1]. Furthermore, over large areas, the path loss related to motion or attenuation in the average signal power corresponds to large-scale fading. A Doppler shift is another characteristic of the wireless channel, and it is generated due to the fact that the wireless communication channel is time varying in nature or due to a movement

* Corresponding author.

E-mail address: eng.awwab.qasim@uobabylon.edu.iq (Awwab Q. Jumaah) 
between the transmitter and receiver [2]. The effect of multipath propagation is clearly seen as shown in Fig. 1, while the transmitted signal arrives at the receiver as multipath signals. They reach at the receiver ends with a various Doppler shift, phase shift, time delay and amplitude.

\section{Multi path propagation effect}

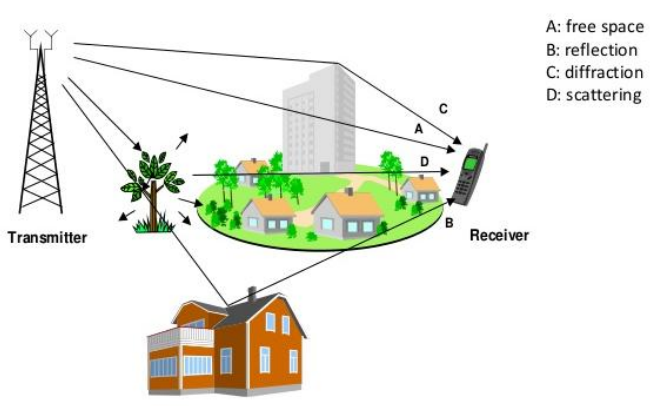

Figure 1: Multipath propagation in mobile communication system

Channel state information (CSI) has to be precisely provided at system receivers to get optimum performance for wireless communication systems and, therefore, transmitted signals can be detected in a coherent way. Otherwise, a non-coherent method is the only way to demodulate transmitted signals such as the differential demodulation technique, result in a loss 3-4 dB in SNR [3]. Many papers have been addressed the problem of channel estimation, and different estimation techniques have been developed to provide Channel state information at the receiver ends so that a reduction in SNR can be mitigated. This paper emphasizes reviewing some recent channel estimation techniques been investigated.

The paper structure is as follows: Wireless channel model is depicted in section 2, while section 3 presents a preview of works that have already been evolved regarding the issue of channel estimation methods. Finally, section 4 gives some concluding remarks of the survey.

\section{Wireless Channel Model}

The wireless communication channel probably characterized by a vector called an impulse response that has most the information to analyze signals transmitted through channels. Basically, the channel impulse response CIR represents the instantaneous case of a dispersive channel due to different multipath components. Resulting, vectors have variable instantaneous amplitudes [4]. Let denote $s(t)$ to be the transmitted signal through a wireless communication, and $x(t)$ to be the equivalent complex baseband form of $s(t)$, which can be described by a multipath fading as:

$s(t)=\operatorname{Re}\left\{x(t) e^{j \omega_{c} t}\right\}$

where $R e\{\cdot\}$ indicates the real part and $\omega_{c}$ is the carrier frequency that equals to $2 \pi f_{c}$. Now, the signal received is formed as:

$r(t)=\sum_{l=0}^{L-1} h_{l}(t) s\left(t-\tau_{l}(t)\right)$

where $L, \tau_{l}(t)$ and $h_{l}(t)$ are the number of paths, $l^{\text {th }}$ path time-variant delay and the complex time-variant amplitude, respectively. Substitute equation (1) into equation (2), yields
$r(t)=\operatorname{Re}\left\{\sum_{l=0}^{L-1} h_{l}(t) e^{-j \omega_{c} \tau_{l}(t)} x\left(t-\tau_{l}(t)\right) e^{j \omega_{c} t}\right\}$

The received signal is assumed now contaminating by a complex additive Gaussian noise $n(t)$ having zero means and equal variances. Those parameters correspond to the parts (real and imaginary) of the noise. Equation (4) shows a mathematical representation of the noise variance:

$\sigma_{n}^{2}=N_{0} B=\mathbb{E}\left\{n(t) n^{*}(t)\right\}$

where $N_{0}$ and $B$ are the power spectral density (W/Hz) and effective bandwidth $(\mathrm{Hz})$ of the noise, respectively. The term $\mathbb{E}\{\cdot\}$ stands for expectation. Equation (3) will be then rewritten after an additive white complex Gaussian noise corrupts the received signal $r(t)$ as [5]:

$y(t)=\sum_{l=0}^{L-1} h_{l}(t) e^{-j \omega_{c} \tau_{l}(t)} x\left(t-\tau_{l}(t)\right)+n(t)$

Let now define $g(t, \tau)$ to be the channel baseband impulse response at the time instant $t$ associated with the multipath fading. Usually, the $\operatorname{CIR} g(t, \tau)$ utilized to model the mobile wireless channels is defined in equation (6). Then the baseband signal $y(t)$ received specified in equation (5) can be rewritten again as in (7).

$g(t, \tau)=\sum_{l=0}^{L-1} h_{l}(t) e^{-j \omega_{c} \tau_{l}(t)} \delta\left(\tau-\tau_{l}(t)\right)$

and

$y(t)=\int_{-\infty}^{\infty} g(t, \tau) x(t-\tau) d \tau+n(t)$

Where $\delta$ is the function of the Dirac Delta. For more simplicity, the linear time-invariant model is assumed to characterize the channel impulse response. This occurs when there is no variation in time $t$ in the second orderof the $g(t, \tau)$ statistics. At this point, a wireless channel considers to be a wide sense stationary (WSS) process. The equation (6) is simplified based on the assumption mentioned to [6]:

$g(\tau)=\sum_{l=0}^{L-1} h_{l} e^{-j \omega_{c} \tau} l \delta\left(\tau-\tau_{l}\right)$

Details of the statistics, sample and non-sample spaced schemes for the CIR can be found in [7, 8, 9 and 10].

\section{The Methods of Channel Estimation}

Signals transmitted via wireless communication are usually distorted: hence, information of any distortion should be necessarily provided using the channel estimation process. Reliability of this process determines performance accuracy of the wireless system, and it can be then used for signal demodulation, decoding or equalization processes [11].This section presents a review of different channel estimation techniques efficiently developed. Decision directed, pilot aided, semi-blind and blind are the essential solving techniques for the problem of channel estimation. Overview for each class is illustrated in the following subsections.

\subsection{Channel Estimation Using Decision Directed Techniques}

The estimation process of this technique employs training symbols along with the detected one. A model of channel estimator using decision directed 
approach is illustrated in Fig. 2 [12]. It is very important to look first on the term of a posteriori channel transfer function. Its initial can be provided based on the obtainable detected symbols, and current received. In the next slot time and throughout the demodulation process of the next received symbols, this a posteriori channel transfer function estimated earlier utilized as an a priori channel estimate [13]. Details of how a block diagram depicted in Fig. 2 works can be found in [14].

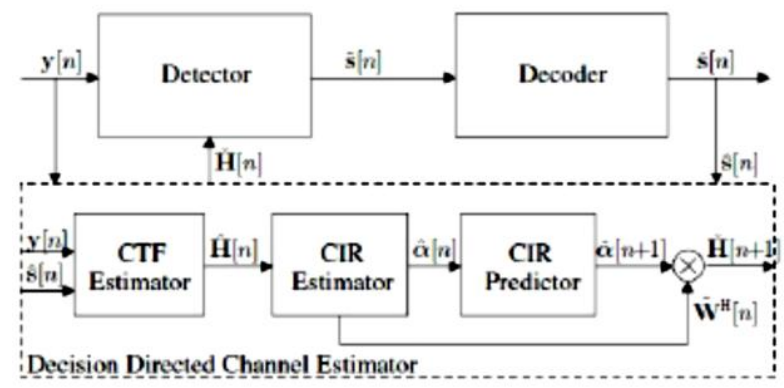

Figure 2: A model of channel estimator using decision directed technique [12]

The important feature of this technique is requiring slight pilot symbols to initialize the process of channel estimation. In [15], Akhtman, and Hanzo proposed decision directed techniques for Code Division Multiple Access (CDMA) having Multi-Carrier and OFDM systems, in which a channel is assumed to be a sample spaced model. In literature, this model is not fit for the real time scenario. However, a scenario of the non-sample spaced model for channel impulse response is assumed to execute the decision directed scheme presented by $[16,17]$ for OFDM systems using the Recursive Least Square (RLS) algorithm as an adaptive predictor [18]. However, the computational complexity is the major drawback when the subcarrier numbers of OFDM system are much more than the channel path numbers. Consequently, Munster and Hanzoin [19] studied the performance of an adaptive OFDM transceiver that uses a modulation mode adaptation and decision directed methods. The multiple input-multiple output (MIMO) OFDM systems restudied as in [20] in order to reduce the inter-antenna interference or inter-symbol interference (ISI). Here, it assumed the channels are independent, and the research effort is to exploit the estimated delay of the channels for estimating channel parameters. Du and Li in [21] exploited a subspace-based decision directed method for MIMO OFDM system using an adaptive filter with low rank. However, at low SNR, it is noted that the performance degrades since the strong noise causes subspace tracking error.

There are two iterative schemes for decision directed channel estimation. A refined hard or soft symbol information determined by a decoder or a detector can be utilized by an estimator and fed it back to achieve high estimation accuracy as an increase in the number of iterations. The hard decision output of the decoder or detector can be used by the estimator if a hard symbol utilized. This is called hard iterative channel estimation. However, if the soft information symbols are employed, the channel estimator makes use of log-likelihood ratios (LLR) on the coded bits determined by either the decoder or detector for channel estimation. This channel iterative scheme is referred to as a soft iterative channel estimation [22].

The hard and soft decision feedbacks taken from equalizer are presented in [23] for iterative channel estimation to improve parameters estimation while the authors in [24] proved that soft decision exhibits better performance than hard decision feedback. Al-Susa and Ormondroyd in [25] discussed a coherent OFDM system utilizing a predictor as a channel estimator in the existence of selective fading channel with the assumption of time-varying frequency. The research effort is exploiting an adaptive technique in a decision directed to make a decision at the output of either the decoder or detector. In [26], a joint iterative channel estimation technique is proposed to build a system receiver under the environment of fading channels by taking the advantage of turbo coding and power of assisted modulation symbols.However, an iterative channel estimation based on a new initial estimation approach of fading channel amplitudes is proposed in [27] as well as a new way of initializing a delayed turbo decoding is presented. Implementation of the Direct Sequence UltraWideband is carried out in [28] that is based on the application of the coherent detection and iterative channel estimation. An improved in Maximum Likelihood (ML) channel estimator is presented by [29] using a decoder of soft input-soft output at the receiver and convolutional encoder at the transmitter. A channel estimator along with the turbo decoder makes an iterative detector that proposed for OFDM systems in [30].Comparative outcomes are illustrated in [31] for soft feedback iterative decision directed technique with turbo codes as well as the channel error correcting coding which is Low-Density Parity Check codes. Lastly, in [32], an iterative decision directed technique having lower complexity for channel estimation of MIMO OFDM systems is presented whilst, in [33], a decision directed approach is proposed for OFDM systems with utilizing fractionally-spaced and sample-spaced channel impulse response estimators.

\subsection{Channel Estimation Using Pilot Assisted Technique}

It is a classical approach for estimating wireless communication channels, and it is known as a training based. The transmitted symbols are multiplexed with the data of training sequences recognized to the receiver at a predetermined position prior transmission. At the receiver end, training symbols are employed to estimate the channel state information (CSI) based on their positions [22].

In the literature, many research works have been published addressing the problem of channel estimation based on the pilot assisted technique. Earlier, in [34], a comparison was done between superimposed pilot assisted modulation schemes, and pilot assisted modulation that was proposed by [35]. It was found that the pilot assisted modulation performs better in bit error rate (BER) than the superimposed pilot assisted modulation technique. However, the opposite results can be achieved in the fast fading channel whereas the tradeoff is high computational complexity. Cai, and Giannakis in [36] proposed a PSAM, which is an adaptive pilot symbol assisted modulation, for solving the problem of both prediction errors and channel estimation. The research effort was maximizing spectral efficiency by optimizing the spacing and power allocation between data symbols and pilots. It was claimed by the authors that, even feedback delay is large, the proposed technique performs well. In [37], the estimator based Maximum likelihood (ML) is investigated for the OFDM system. In fast varying channels, the article in [38] proposed a pseudo pilot algorithm, which mainly depends on the approach of regression model based least squares fitting, for detecting data symbols without increasing the pilot density. Moreover, the authors in [39] present a pilot pattern design and an optimal training for OFDM systems over a Rayleigh fading channel. In [40], Pilotassisted channel estimation is optimized for open loop OFDM systems. It is assumed optimal pilots have uniform spacing. Further, to minimize feedback, Lloyd algorithm and vector quantization together are used. In 
[41], a comparison between the Bayesian minimum mean squared error (BMMSE), which exploits a prior of informational statistics about the channel, and the ML estimators is done for OFDM systems. Since ML estimator does not necessitate any of the channel informational statistics, its implantation is very simple. However, it exhibits performance degradation at low SNR while BMMSE exhibits better performance due to a prior of information statistics. They have comparable performance at high SNR.

Finally, many research papers have been published with the system consideration of MIMO regards to the technique of pilot assisted channel estimation. Such contribution found in [42 and 43]. In [44], the authors presented a design of MIMO OFDM systems with optimal training in the presence of phase noise and frequency offset.

\subsection{Channel Estimation Using Semi-blind and Blind Techniques}

Training symbols do not require in this technique, while the observed received signal, which is the only measureable signal, used for estimating channels by providing the transmitted signal properties and inherent information. The blind techniques can be classified into statistical approaches that utilize the cyclic properties of signals received and deterministic methods that assume or consider channel parameters and signals received having deterministic quantities [45]. In the literature, blind channel estimation using deterministic and statistical methods can be found in [46-48] for single antenna OFDM communication systems. The process of blind channel estimation is depicted in Fig. 3.

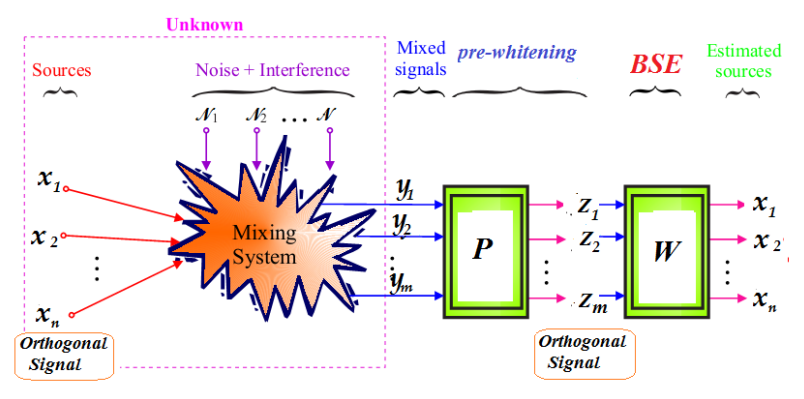

Figure 3: Simple block Diagram of the process of blind channel estimation [49]

The higher order statistics (HOS) of received signals are what earlier blind methods depend on for estimate channel coefficients. Such works found in [50-51]. A high computational complexity faces them since these methods require a long record of data samples or huge information. Therefore, researchers relied on the second order cyclic statistics (SOS) to invent and develop new blind methods as in [52 and 53]. In [54], the channel estimation based blind method is proposed in the frequency domain whereas, in [55], the single input-single output (SISO) FIR channel is blindly estimated by using SOS of transformed data. Necker and Stuber in [56] studied deterministic blind approach based on the ML for phase shift keying signals. In OFDM communication systems, many research papers have addressed blind estimation approaches using subspace schemes [5759].In [60], a blind channel estimation technique is proposed for un-coded OFDM. It is used a constrained linear minimum mean square error (MMSE) to estimate an initial data symbol for each subcarrier. The authors claim that the blind system proposed is very favorable, and it can be utilized for high
Doppler. In [61] however, the authors utilize subspace method to blindly estimate channel for MIMO OFDM systems, and in [62], subspace based MIMO OFDM system is employed for blind channel estimation considering short averaging periods. Different blind estimation techniques that are exploited HOS of received signals are presented in [63-65] for MIMO systems. Furthermore, Alamouti STBC $(2 \times 2)$ channel with QPSK modulation scheme is studied to estimate DOA based on the technique of independent component analysis (ICA)[66].

On the other hand, the estimation techniques based semi-blind require, for channel estimation purpose, known pilot symbols in a combination with the inherent information obtained in the unknown received signals. In [67], the semi-blind approaches are presented using first order statistics to estimate channel coefficients based on superimposing periodic pilot sequences. In OFDM with the space-time block (STB) pre-coded systems as in [68], semi-blind channel estimation is employed. In [69], the semi-blind estimation method is suggested in the case of frequency selective MIMO systems. Finally, semi-blind channel estimation employing an orthogonal pilot based ML is proposed in [70]. The channel matrix in this approach is factored into a unitary rotation matrix that can be estimated using an orthogonal pilot based ML from the training symbols and a whitening matrix that estimated using blind algorithms.

\section{Conclusions}

A review of fading channel and different channel estimation techniques include; decision directed, the pilot assisted, blind and semi-blind techniques is presented in this paper. Variant and invariant multipath CIR models are also given in a simple mathematical way. Although the blind techniques are very efficient, and training symbols are not required, ahigh computational complexity is a major drawback. Consequently, they applied to time-invariant channel and limited to slowly time-variant channel. Although the technique of pilot assisted is simple in implementation, it suffers from two main drawbacks. The major drawback, in general, is communication bandwidth wastage. The second one is that this technique depends mainly on the pilot symbols and data points, estimated by interpolation techniques that in reality have not an ideal performance, cannot be determined a hundred percent. Hence, during the estimation processes, unresolved errors would be presented. However, the semi-blind techniques using for channel estimation problem perform better than the blind methods and pilot assisted techniques. Finally, the decision direct channel estimation techniques with their iterative schemes outperform than the semi-blind, blind and pilot assisted channel estimation techniques.

\section{REFERENCES}

1. Sklar, Bernard. "Rayleigh fading channels in mobile digital communication systems. I. Characterization." IEEE Communications Magazine 35.7 (1997): 90-100. 2. Sayeed, Akbar M., and BehnaamAazhang. "Joint multipath-Doppler diversity in mobile wireless communications." IEEE Transactions on Communications 47.1 (1999): 123-132.

3. Li, Ye Geoffrey, Leonard J. Cimini, and Nelson R. Sollenberger. "Robust channel estimation for OFDM systems with rapid dispersive fading channels." Communications, 1998. ICC 98. Conference Record. 1998 IEEE International Conference on. Vol. 3. IEEE, 1998.

4. Prasad, Ramjee. OFDM for wireless communications systems. Artech House, 2004.

5. Ibnkahla, Mohamed, ed. Signal processing for mobile communications handbook. CRC press, 2004.

6. Hanzo, Lajos, et al. OFDM and MC-CDMA for broadband multi-user communications, WLANs and broadcasting. John Wiley \& Sons, 2005. 
7. Rappaport, Theodore S. "Wireless Communications--Principles and Practice, (The Book End)." Microwave Journal 45.12 (2002): 128-129.

8. Althahab, AwwabQasim. Performance analysis of adaptive blind equalization algorithms for noisy FIR and IIR channels. University of Colorado at Denver, 2013.

9. Kuhn, Volker. Wireless communications over MIMO channels: applications to CDMA and multiple antenna systems. John Wiley \& Sons, 2006.

10. Oyerinde, Olutayo O., and Stanley H. Mneney. "Review of channel estimation for wireless communication systems." IETE Technical review 29.4 (2012): 282-298.

11. Chiueh, Tzi-Dar, and Pei-Yun Tsai. OFDM baseband receiver design for wireless communications. John Wiley \& Sons, 2008.

12. Hanzo, Lajos, et al. MIMO-OFDM for LTE, WiFi and WiMAX: Coherent versus non-coherent and cooperative turbo transceivers. John Wiley \& Sons, 2010.

13. Bernard. Harris Sklar (Fredric J.). Digital Communications: Fundamentals and Applications. Pearson Education Limited, 2018.

14. Madni, Haji Muhammad Furqan Ahmed, Bakhtiar Ali, and Muhammad Fasih Uddin Butt. "Study and Analysis of Channel Estimation Techniques for OFDM Based Wireless Communication Systems." Frontiers of Information Technology (FIT), 2014 12th International Conference on. IEEE, 2014.

15. Akhtman, J., and L. Hanzo. "Generic reduced-complexity MMSE channel estimation for OFDM and MC-CDMA." Vehicular Technology Conference, 2005. VTC 2005-Spring. 2005 IEEE 61st. Vol. 1. IEEE, 2005.

16. Akhtman, J., and L. Hanzo. "Decision directed channel estimation employing approximation subspace tracking." Proceedings of Spring 5 (2007): 305660.

17. Akhtman, Jos, and Lajos Hanzo. "Decision directed channel estimation aided OFDM employing sample-spaced and fractionally-spaced CIR estimators." IEEE Transactions on Wireless Communications 6.4 (2007).

18. Schafhuber, Dieter, and Gerald Matz. "MMSE and adaptive prediction of time-varying channels for OFDM systems." IEEE transactions on wireless communications 4.2 (2005): 593-602.

19. Munster, M., and Lajos Hanzo. "MMSE channel prediction assisted symbol-by-symbol adaptive OFDM." Communications, 2002. ICC 2002. IEEE International Conference on. Vol. 1. IEEE, 2002.

$20 . \quad$ Li, Y. Geoffrey, Jack H. Winters, and Nelson R. Sollenberger. "MIMOOFDM for wireless communications: signal detection with enhanced channel estimation." IEEE Transactions on Communications 50.9 (2002): 1471-1477.

21. Du, Jianxuan, and Ye Li. "MIMO-OFDM channel estimation based on subspace tracking." Vehicular Technology Conference, 2003. VTC 2003-Spring. The 57th IEEE Semiannual. Vol. 2. IEEE, 2003.

22. John G..Proakis, and MasoudSalehi. Digital communications.5th Edition. McGraw-Hill, 2008.

23. Sandell, Magnus, et al. "Iterative channel estimation using soft decision feedback." Global Telecommunications Conference, 1998. GLOBECOM 1998. The Bridge to Global Integration. IEEE. Vol. 6. IEEE, 1998.

24. Kobayashi, Mari, Joseph Boutros, and Giuseppe Caire. "Successive interference cancellation with SISO decoding and EM channel estimation." IEEE Journal on selected areas in Communications 19.8 (2001): 1450-1460.

25. Al-Susa, E., and R. F. Ormondroyd. "A predictor-based decision feedback channel estimation method for COFDM with high resilience to rapid timevariations." Vehicular Technology Conference, 1999. VTC 1999-Fall. IEEE VTS 50th. Vol. 1. IEEE, 1999.

26. Li, Qinghua, Costas N. Georghiades, and Xiaodong Wang. "An iterative receiver for turbo-coded pilot-assisted modulation in fading channels." IEEE Communications letters 5.4 (2001): 145-147.

27. Mielczarek, Bartosz, and Arne Svensson. "Improved iterative channel estimation and turbo decoding over flat-fading channels." Vehicular Technology Conference, 2002. Proceedings. VTC 2002-Fall. 2002 IEEE 56th. Vol. 2. IEEE, 2002. 28. Son, Le Thanh, and K. M. Ahmed. "Performance of coherent direct sequence ultra wideband receiver with iterative channel estimation and detection." Communications and Information Technology, 2004. ISCIT 2004. IEEE International Symposium on. Vol. 2. IEEE, 2004.

29. Li, Yabo, et al. "Coding assisted iterative channel estimation for impulse radio ultra-wide band communication systems." Acoustics, Speech, and Signal Processing, 2005. Proceedings.(ICASSP'05). IEEE International Conference on. Vol. 3. IEEE, 2005.

30. Ammari, Mohamed L., and Francois Gagnon. "Iterative channel estimation and decoding of turbo-coded OFDM symbols in selective Rayleigh channel." Canadian Journal of Electrical and Computer Engineering 32.1 (2007): 918.
31. Beinschob, P., M. Lieberei, and U. Zölzer. "Improving MIMO-OFDM decision-directed channel estimation by utilizing error-correcting codes." Advances in Radio Science 7.C. 2 (2009): 83-88.

32. Oyerinde, OlutayoOyeyemi, and Stanley H. Mneney. "Iterative decision directed channel estimation for BICM-based MIMO-OFDM systems." Communications (ICC), 2010 IEEE International Conference on. IEEE, 2010.

33. Akhtman, Jos, and Lajos Hanzo. "Decision directed channel estimation aided OFDM employing sample-spaced and fractionally-spaced CIR estimators." IEEE Transactions on Wireless Communications 6.4 (2007).

34. Cavers, James K., and Maria Liao. "A comparison of pilot tone and pilot symbol techniques for digital mobile communication." Global Telecommunications Conference, 1992. Conference Record., GLOBECOM'92. Communication for Global Users., IEEE. IEEE, 1992.

35. Sampei, Seiichi, and Terumi Sunaga. "Rayleigh fading compensation method for 16QAM in digital land mobile radio channels." Vehicular Technology Conference, 1989, IEEE 39th. IEEE, 1989.

36. Cai, Xiaodong, and Georgios B. Giannakis. "Adaptive PSAM accounting for channel estimation and prediction errors." IEEE Transactions on Wireless Communications 4.1 (2005): 246-256.

37. Negi, Rohit, and John Cioffi. "Pilot tone selection for channel estimation in a mobile OFDM system." IEEE Transactions on Consumer Electronics 44.3 (1998): 1122-1128.

38. Chang, Ming-Xian, and Tsung-Da Hsieh. "Detection of OFDM signals in fast-varying channels with low-density pilot symbols." IEEE Transactions on Vehicular Technology 57.2 (2008): 859-872.

39. Zhang, Wei, Xiang-Gen Xia, and Pak-Chung Ching. "Optimal training and pilot pattern design for OFDM systems in Rayleigh fading." IEEE Transactions on Broadcasting 52.4 (2006): 505-514.

40. Panah, Ali Y., Rodney G. Vaughan, and Robert W. HeathJr. "Optimizing pilot locations using feedback in OFDM systems." IEEE Transactions on Vehicular Technology 58.6 (2009): 2803-2814.

41. Morelli, Michele, and Umberto Mengali. "A comparison of pilot-aided channel estimation methods for OFDM systems." IEEE Transactions on signal processing 49.12 (2001): 3065-3073.

42. Jungnickel, Volker, et al. "Performance of a MIMO system with overlay pilots." Global Telecommunications Conference, 2001. GLOBECOM'01. IEEE. Vol. 1. IEEE, 2001.

43. Jeon, Won Gi, Kyung Hyun Paik, and Yong Soo Cho. "An efficient channel estimation technique for OFDM systems with transmitter diversity." IEICE Transactions on Communications 84.4 (2001): 967-974.

44. Minn, Hlaing, and Naofal Al-Dhahir. "Optimal training signals for MIMO OFDM channel estimation." IEEE transactions on wireless communications 5.5 (2006): $1158-1168$.

45. Petropulu, Athina, Ruifeng Zhang, and Rui Lin. "Blind OFDM channel estimation through simple linear precoding." IEEE Transactions on Wireless Communications 3.2 (2004): 647-655.

46. Liu, Hui, Guanghan Xu, and Lang Tong. "A deterministic approach to blind equalization." Signals, Systems and Computers, 1993. 1993 Conference Record of The Twenty-Seventh Asilomar Conference on. IEEE, 1993.

47. Chen, S., and S. McLaughlin. "Blind channel identification based on higher-order cumulant fitting using genetic algorithms." Higher-Order Statistics, 1997., Proceedings of the IEEE Signal Processing Workshop on. IEEE, 1997.

48. Muquet, Bertrand, and Marc de Courville. "Blind and semi-blind channel identification methods using second order statistics for OFDM systems." Acoustics, Speech, and Signal Processing, 1999. Proceedings., 1999 IEEE International Conference on. Vol. 5. IEEE, 1999.

49. Althahab, AwwabQasimJumaah and Alrufaiat, Sameer Abdul Kadhim." A Comprehensive Review on Various Estimation Techniques for Multi Input Multi Output Channel." Journal of University of Babylon for Engineering Sciences 27.1 (2019): $262-274$

50. Shalvi, Ofir, and Ehud Weinstein. "New criteria for blind deconvolution of nonminimum phase systems (channels)." IEEE Transactions on information theory 36.2 (1990): 312-321.

51. Petropulu, Athina P., and Chrysostomos L. Nikias. "Blind convolution using signal reconstruction from partial higher order cepstral information." IEEE transactions on signal processing 41.6 (1993): 2088-2095.

52. Tong, L. "Blind identification and equalization using spectral measures, part II: A time domain approach." Cyclostationarity in Communication and Signal Processing (1993): 437-454.

53. Zeng, Hanks H., and Lang Tong. "Blind channel estimation using the second-order statistics: algorithms." IEEE Transactions on Signal Processing 45.8 (1997): 1919-1930. 
54. Yatawatta, Sarod, Athina P. Petropulu, and RiddhiDattani. "Blind channel estimation using fractional sampling." IEEE transactions on vehicular technology 53.2 (2004): 363-371.

55. Delmas, Jean Pierre, Yann Meurisse, and Pierre Comon. "Performance limits of alphabet diversities for FIR SISO channel identification." IEEE Transactions on Signal Processing 57.1 (2009): 73-82.

56. Necker, Marc C., and Gordon L. Stuber. "Totally blind channel estimation for OFDM over fast varying mobile channels." Communications, 2002. ICC 2002. IEEE International Conference on. Vol. 1. IEEE, 2002.

57. Cai, Xiaodong, and Ali N. Akansu. "A subspace method for blind channel identification in OFDM systems." Communications, 2000. ICC 2000. 2000 IEEE International Conference on. Vol. 2. IEEE, 2000.

58. Muquet, Bertrand, Marc De Courville, and Pierre Duhamel. "Subspacebased blind and semi-blind channel estimation for OFDM systems." IEEE Transactions on signal processing 50.7 (2002): 1699-1712.

59. Doukopoulos, Xenofon G., and George V. Moustakides. "Blind adaptive channel estimation in OFDM systems." IEEE Transactions on Wireless Communications 5.7 (2006): 1716-1725.

60. Banani, SeyedAlireza, and Rodney G. Vaughan. "OFDM with iterative blind channel estimation." IEEE Transactions on Vehicular Technology 59.9 (2010): 4298-4308.

61. Zhou, Shengli, Bertrand Muquet, and Georgios B. Giannakis. "Subspacebased (semi-) blind channel estimation for block precoded space-time OFDM." IEEE Transactions on Signal Processing 50.5 (2002): 1215-1228.

62. Tu, Chao-Cheng, and Benoit Champagne. "Subspace blind MIMOOFDM channel estimation with short averaging periods: Performance analysis." Wireless Communications and Networking Conference, 2008. WCNC 2008. IEEE.
IEEE, 2008.

63. Tugnait, Jitendra K. "Identification and deconvolution of multichannel linear non-Gaussian processes using higher order statistics and inverse filter criteria." IEEE Transactions on Signal Processing 45.3 (1997): 658-672.

64. Chen, Binning, and Athina P. Petropulu. "Frequency domain blind MIMO system identification based on second-and higher order statistics." IEEE Transactions on Signal Processing 49.8 (2001): 1677-1688.

65. Acar, Turev, Yuanning Yu, and Athina P. Petropulu. "Blind MIMO system estimation based on PARAFAC decomposition of higher order output tensors." IEEE Transactions on Signal Processing 54.11 (2006): 4156-4168.

66. Alrufaiaat, Sameer Abdul Kadhim, and AwwabQasimJumaahAlthahab. "A new DOA estimation approach for QPSK Alamouti STBC using ICA technique- based PSO algorithm." International Journal of Communication Systems 32.2 (2019): e3858. 67. Zhou, G. Tong, Mats Viberg, and Tomas McKelvey. "A first-order statistical method for channel estimation." IEEE signal processing letters 10.3 (2003): $57-60$.

68. Zhou, Shengli, Bertrand Muquet, and Georgios B. Giannakis. "Semiblind channel estimation for block precoded space-time OFDM transmissions." Statistical Signal Processing, 2001. Proceedings of the 11th IEEE Signal Processing Workshop on. IEEE, 2001.

69. Wo, Tianbin, and Peter Adam Hoeher. "Semi-blind channel estimation for frequency-selective MIMO systems." Proc. 14-th IST Mobile \& Wireless Communications Summit, Dresden, Germany (2005).

70. Jagannatham, Aditya K., and Bhaskar D. Rao. "Whitening-rotation-based semi-blind MIMO channel estimation." IEEE Transactions on Signal Processing 54.3 (2006): 861-869. 